Research Article

\title{
Thermodynamics in $f(T)$ Gravity with Nonminimal Coupling to Matter
}

\author{
Tahereh Azizi and Najibeh Borhani \\ Department of Physics, Faculty of Basic Sciences, University of Mazandaran, Babolsar 47416 95447, Iran \\ Correspondence should be addressed to Tahereh Azizi; t.azizi@umz.ac.ir
}

Received 17 September 2016; Revised 28 November 2016; Accepted 28 December 2016; Published 17 January 2017

Academic Editor: Chao-Qiang Geng

Copyright ( 2017 Tahereh Azizi and Najibeh Borhani. This is an open access article distributed under the Creative Commons Attribution License, which permits unrestricted use, distribution, and reproduction in any medium, provided the original work is properly cited. The publication of this article was funded by SCOAP ${ }^{3}$.

\begin{abstract}
In the present paper, we study the thermodynamics behavior of the field equations for the generalized $f(T)$ gravity with arbitrary coupling between matter and the torsion scalar. In this regard, we explore the verification of the first law of thermodynamics at the apparent horizon of the Friedmann-Robertson-Walker universe in two different perspectives, namely, the nonequilibrium and equilibrium descriptions of thermodynamics. Furthermore, we investigate the validity of the second law of thermodynamics for both descriptions of this scenario with the assumption that the temperature of matter inside the horizon is similar to that of horizon.
\end{abstract}

\section{Introduction}

The teleparallel equivalent of general relativity (TEGR) [1,2] is an equivalent formulation of classical gravity, which, instead of using the curvature defined via the Levi-Civita connection, uses the Weitzenböck connection that has no curvature but only torsion. This approach is closely related to the standard general relativity, differing only in "boundary terms" involving total derivatives in the action. In this setup, the dynamical objects are the four linearly independent vierbeins and the Lagrangian density, $T$, is constructed from the torsion tensor which is formed solely from products of the first derivatives of the vierbein [2]. However, in a similar manner to the $f(R)$ modified gravity [3-7], the teleparallel gravity is generalized to a modified $f(T)$ version [8-11]. The Lagrangian density is an arbitrary function of the torsion scalar $T$. This modification enables the theory to explain the late-time acceleration of the universe $[9,12,13]$ which is favored by the observational data. So there is no need to introduce a mysterious dark energy component for the matter content of the universe. The significant advantage of $f(T)$ gravity is that the field equations are second-order differential equations and are more manageable compared to $f(R)$ theories. For some gravitational and cosmological aspects of the modified teleparallel gravity, see [14]. Recently, further generalization of the teleparallel gravity has been introduced in [15] by considering nonminimal coupling between matter and the torsion scalar in the action. In this model, the gravitational field can be described in terms of two arbitrary functions of the torsion scalar $T$, namely, $f_{1}(T)$ and $f_{2}(T)$, with the function $f_{2}(T)$ linearly coupled to the matter Lagrangian [15]. This nonminimal torsion-matter coupling scenario can offer a unified description of the universe evolution from its inflationary to the late-time accelerated phases [15]. In [16], the energy conditions of this model are studied and the validity of energy bounds is examined. The dynamical system analysis for the cosmological applications of this model is carried out in [17].

In the present work, we are going to study the thermodynamics aspects of this nonminimally coupled $f(T)$ model at the apparent horizon of an expanding cosmological background. Indeed, the black hole thermodynamics sets up connections between general relativity and the laws of thermodynamics [18]. In this content, a temperature and entropy, which are proportional to the surface gravity and area of the horizon, respectively, are associated with the black hole. The first law of black hole thermodynamics is given by the identity $T d S=d M$ [19], where $M$ is the mass of the black hole. Furthermore, Jacobson [20] showed that Einstein's equations can be derived from the fundamental relation $d Q=T d S$ 
in all local Rindler horizons, where $\delta Q$ and $T$ are the energy flux across the horizon and Unruh temperature, respectively. This approach soon generalized to the cosmological situation, where it was shown that, by applying the Clausius relation to the apparent horizon of the Friedmann-Robertson-Walker (FRW) universe, the Friedmann equation can be rewritten in the form of the first law of thermodynamics [21]. Recently, the equivalence of the Clausius relation and the gravitational field equations has been investigated to the more general modified theories of gravity such as Gauss-Bonnet gravity [22], Lovelock gravity [23, 24], Braneworld gravity [25], scalar-tensor gravity [26], $f(R)$ theories [27-31], and the extended models of $f(R)$-gravity [32-37]. In the context of $f(T)$ gravity, the first law of black hole thermodynamics has been studied in [38] and the thermodynamics of the apparent horizon of the FRW universe is explored in [39-41]. This issue is also studied in some modified $f(T)$ scenarios [42-45].

On the other hand, in [27], it was pointed out that, in order to derive the field equations of $f(R)$ modified gravity, one should employ a nonequilibrium thermodynamics treatment. However, it has been demonstrated in [29] that it is possible to obtain an equilibrium description of thermodynamics on the apparent horizon of $f(R)$ gravity. The same works also have been carried out in $f(T)$ gravity [40] and some extended models of $f(R)$ gravity $[29,37]$. In addition to the first law of thermodynamics, there has been a lot of interest in exploring the second law of thermodynamics in gravitational theories [46-54]. According to the second law of thermodynamics, the sum of the horizon entropy and the entropy of the matter field, that is, the total entropy, is a nondecreasing function of time.

In this paper, we explore the laws of thermodynamics in both nonequilibrium and equilibrium descriptions in the nonminimal $f(T)$ gravity model. The organization of the paper is as follows. In Section 2, we briefly review the nonminimally torsion-matter coupling model and its equations of motion. In Section 3, we treat nonequilibrium descriptions of thermodynamics and investigate the first and second laws of thermodynamics. We explore the equilibrium description of thermodynamics in Section 4. Finally, our conclusion will appear in Section 5.

\section{The Equations of Motion}

In the context of the teleparallel gravity, the dynamical object is a vierbein field $e_{i}\left(x^{\mu}\right), i=0,1,2,3$, which is an orthonormal basis for the tangent space at each point $x^{\mu}$ of the manifold. The metric tensor is obtained from the dual vierbein as $g_{\mu \nu}(x)=\eta_{i j} e_{\mu}^{i}(x) e_{\nu}^{j}(x)$, where $\eta_{i j}=e_{i} \cdot e_{j}$ is the Minkowski metric and $e_{i}^{\mu}$ is the component of the vector $e_{i}$ in a coordinate basis. Note that the Greek indices label coordinates on the manifold, while Latin indices refer to the tangent space. The torsion tensor is defined as

$$
T_{\mu \nu}^{\lambda}=e_{i}^{\lambda}\left(\partial_{\mu} e_{\nu}^{i}-\partial_{\nu} e_{\mu}^{i}\right)
$$

Defining other two tensors,

$$
\begin{aligned}
S_{\lambda}{ }^{\mu \nu} & \equiv \frac{1}{2}\left(K_{\lambda}^{\mu \nu}+\delta_{\lambda}^{\mu} T_{\sigma}^{\sigma \nu}-\delta_{\lambda}^{\nu} T_{\sigma}^{\sigma \mu}\right), \\
K_{\lambda}^{\mu \nu} & \equiv-\frac{1}{2}\left(T_{\lambda}^{\mu \nu}-T_{\lambda}^{\nu \mu}-T_{\lambda}^{\mu \nu}\right),
\end{aligned}
$$

one can write down the torsion scalar $T \equiv S_{\lambda}{ }^{\mu \nu} T^{\lambda}{ }_{\nu \mu}$. Using the torsion scalar as the teleparallel Lagrangian leads to the same gravitational equations of the general relativity. In this work, we focus on the modified teleparallel gravity with nonminimal coupling between the torsion scalar and the matter Lagrangian which is introduced via the following action [15]:

$$
S=\frac{1}{16 \pi G} \int d^{4} x|e|\left\{T+f_{1}(T)+\left[1+\lambda f_{2}(T)\right] \mathscr{L}_{m}\right\},
$$

where $e=\operatorname{det}\left(e_{\mu}^{i}\right)=\sqrt{-g}, f_{1}(T)$ and $f_{2}(T)$ are arbitrary functions of the torsion scalar, and $\lambda$ is a coupling constant with units of mass ${ }^{-2}$. Varying the action with respect to the vierbein leads to the field equations [15]:

$$
\begin{aligned}
(1+ & \left.F_{1}+\lambda F_{2} \mathscr{L}_{m}\right)\left[e^{-1} \partial_{\mu}\left(e e_{A}^{\alpha} S_{\alpha}{ }^{\rho \mu}\right)-e_{A}^{\alpha} T^{\mu}{ }_{\nu \alpha} S_{\mu}^{\nu \rho}\right] \\
& +\left(F_{1}^{\prime}+\lambda F_{2}^{\prime} \mathscr{L}_{m}\right) \partial_{\mu} T e_{A}^{\alpha} S_{\alpha}{ }^{\rho \mu}+\frac{1}{4} e_{A}^{\rho}\left(f_{1}+T\right) \\
& -\frac{1}{4} \lambda F_{2} \partial_{\mu} T e_{A}^{\alpha} S_{\alpha}^{\text {em }}{ }^{\rho \mu}+\lambda F_{2} e_{A}^{\alpha} S_{\alpha}{ }^{\rho \mu} \partial_{\mu} \mathscr{L}_{m} \\
= & 4 \pi G\left(1+\lambda f_{2}\right) e_{A}^{\alpha} T_{\alpha}^{\text {em }}{ }^{\rho},
\end{aligned}
$$

where $F_{i}=d f_{i} / d T$ and the prime denotes a derivative with respect to the torsion scalar and we have defined ${ }_{S}^{\mathrm{em}}{ }_{A}{ }^{\rho \mu}=$ $\partial \mathscr{L}_{m} / \partial \partial_{\mu} e_{\rho}^{A}$. We assume that the matter content of the universe is given by a perfect fluid and the matter Lagrangian density is described by $\mathscr{L}_{m}=-\rho_{m}$ which leads to $S_{A}^{\mathrm{em} \rho \mu}=0$. So the energy momentum tensor of the matter is given by

$$
\stackrel{\mathrm{em}}{T}_{\mu \nu}=\left(\rho_{m}+p_{m}\right) u_{\mu} u_{\nu}-p_{m} g_{\mu \nu},
$$

where $u^{\mu}$ is the four velocities of the fluid in the comoving coordinates. For a flat homogeneous and isotropic FriedmanRobertson-Walker (FRW) universe, the vierbein is given by

$$
e_{\mu}^{i}=\operatorname{diag}[1, a(t), a(t), a(t)],
$$

where $a(t)$ is the cosmological scale factor. Using the above relation together with (1) and (2), one obtains $T=-6 H^{2}$, where $H=\dot{a} / a$ is the Hubble parameter. The substitution of the FRW vierbein (6) in the field equation (4) yields the modified Friedmann equations as follows:

$$
\begin{aligned}
H^{2} & =\frac{1}{3 \mathscr{F}}\left[8 \pi G\left(1+\lambda f_{2}\right) \rho_{m}-\frac{f_{1}}{2}\right], \\
\dot{H} & =-\frac{1}{\mathscr{F}}\left[4 \pi G\left(\rho_{m}+P_{m}\right)\left[1+\lambda\left(f_{2}-2 T F_{2}\right)\right]\right. \\
& \left.+H\left(\dot{F}_{1}-16 \pi G \lambda \rho_{m} \dot{F}_{2}\right)\right],
\end{aligned}
$$


where $\dot{F}_{i}=d F_{i} / d t$ and $\mathscr{F}=\left(1+2 F_{1}-32 \pi G \lambda \rho_{m} F_{2}\right)$. Note that the usual $f(T)$ gravity can be recovered in the limit $\lambda=0$. It has been shown that (7) can describe the acceleration expansion of the universe without introduction of any dark energy component [15]. In the rest of this paper, we concentrate on the thermodynamic aspects of the nonminimal torsion-matter coupling extension of teleparallel gravity.

\section{Nonequilibrium Picture}

To study the thermodynamics of the nonminimal $f(T)$ gravity, we rewrite (7) as follows:

$$
\begin{aligned}
H^{2} & =\frac{8 \pi G}{3 \mathscr{F}}\left(\hat{\rho}_{d}+\rho_{m}\right), \\
\dot{H} & =-\frac{4 \pi G}{\mathscr{F}}\left(\hat{\rho}_{d}+\widehat{p}_{d}+\rho_{m}+p_{m}\right),
\end{aligned}
$$

where the energy density and pressure of the dark components are defined as

$$
\begin{aligned}
\hat{\rho}_{d} & \equiv \frac{1}{16 \pi G}\left(T F_{1}-f_{1}\right)+\lambda \rho_{m}\left(f_{2}-T F_{2}\right), \\
\widehat{p}_{d} & \equiv \frac{1}{16 \pi G}\left[f_{1}-T F_{1}+4 H\left(\dot{F}_{1}-16 \pi G \lambda \rho_{m} \dot{F}_{2}\right)\right. \\
& \left.+\lambda P_{m}\left(f_{2}-2 T F_{2}\right)-\lambda \rho_{m} T F_{2}\right],
\end{aligned}
$$

respectively. Here, a hat denotes quantities in the nonequilibrium description of thermodynamics which do not satisfy the standard continuity equation, so that

$$
\begin{aligned}
\dot{\hat{\rho}}_{d}+3 H\left(\widehat{\rho}_{d}+\widehat{p}_{d}\right)= & \frac{1}{16 \pi G}\left(-T \dot{F}_{1}\right)+\lambda \rho_{m} T \dot{F}_{2} \\
& -3 \lambda T H F_{2}\left(\rho_{m}+p_{m}\right) .
\end{aligned}
$$

The perfect fluid satisfies the continuity equations by virtue of the Bianchi identity:

$$
\dot{\rho}_{m}+3 H\left(\rho_{m}+p_{m}\right)=0 .
$$

3.1. First Law of Thermodynamics. Now we investigate the thermodynamic behavior of the nonminimal $f(T)$ gravity on the apparent horizon. In the flat FRW universe, the radius $\tilde{r}_{A}$ of the dynamical apparent horizon is given by [22]

$$
\tilde{r}_{A}=\frac{1}{H},
$$

By taking the time derivative of this equation and substituting (10) into the result, we obtain

$$
\frac{\mathscr{F}}{4 \pi G} d \widetilde{r}_{A}=H \widetilde{r}_{A}^{3}\left(\widehat{\rho}_{d}+\widehat{p}_{d}+\rho_{m}+p_{m}\right) d t .
$$

In the Einstein gravity, the Bekenstein-Hawking relation $S=$ $A /(4 G)$ defines the horizon entropy, where $A=4 \pi \widetilde{r}_{A}^{2}$ is the area of the apparent horizon [55]. In the framework of the generalized theories of gravity such as $f(R)$ modified gravity, a horizon entropy $\widehat{S}$ associated with a Noether charge, called the Wald entropy [56], is expressed as $\widehat{S}=A /\left(4 G_{\text {eff }}\right)$, where $G_{\text {eff }}$ is the effective gravitational coupling [57].

In the context of $f(T)$ gravity, it has been shown that the first law of black hole thermodynamics breaks down [38] due to the violation of local Lorentz invariance [58]. However, it is argued that when $f^{\prime \prime}=d^{2} f / d T^{2}$ is small, the entropy of the black hole in $f(T)$ gravity is approximately equal to $f^{\prime}(T) A / 4$. Furthermore, from the study of the matter density perturbations in $f(T)$ gravity, one can take the effective gravitational coupling taken as $G_{\text {eff }}=G / f^{\prime}(T)$. Hence, similar to $f(T)$ case, with the Friedmann equation (10), we take the effective gravitational coupling as $G_{\text {eff }}=G / \mathscr{F}$, so the Wald entropy in nonminimal $f(T)$ gravity is given by

$$
\widehat{S}=\frac{\mathscr{F} A}{4 G} .
$$

Using (16) and (17), we find

$$
\frac{1}{2 \pi \widetilde{r}_{A}} d \widehat{S}=4 \pi \widetilde{r}_{A}^{3} H\left(\widehat{\rho}_{d}+\widehat{p}_{d}+\rho_{m}+p_{m}\right) d t+\frac{\widetilde{r}_{A}}{2 G} \dot{\mathscr{F}} d t .
$$

The temperature of the apparent horizon is given by the Hawking temperature $T_{h}=\left|\kappa_{s}\right| / 2 \pi$, where $\kappa_{s}=-\left(1 / \widetilde{r}_{A}\right)(1-$ $\left.\dot{\vec{r}}_{A} / 2 H \widetilde{r}_{A}\right)$ is the surface gravity at the apparent horizon. Multiplying (18) with the term $1-\dot{\tilde{r}}_{A} /\left(2 H \widetilde{r}_{A}\right)$ yields

$$
\begin{aligned}
T_{h} d \widehat{S}= & 4 \pi \widetilde{r}_{A}^{3} H\left(\widehat{\rho}_{d}+\widehat{p}_{d}+\rho_{m}+p_{m}\right) d t \\
& -2 \pi \widetilde{r}_{A}^{2}\left(\widehat{\rho}_{d}+\widehat{p}_{d}+\rho_{m}+p_{m}\right) d \widetilde{r}_{A} \\
& +\frac{\pi \widetilde{r}_{A}^{2} T_{h}}{G} d \mathscr{F} .
\end{aligned}
$$

In the Einstein gravity, the total energy inside a sphere of radius $\widetilde{r}_{A}$ of the apparent horizon is $E=\widetilde{r}_{A} / 2 G$. However, in the context of generalized gravity, one should use the effective gravitational constant in this relation. Hence, in the nonminimal modified $f(T)$ gravity, the total energy is given by the following equation:

$$
\widehat{E}=\frac{\widetilde{r}_{A} \mathscr{F}}{2 G}=\frac{3 V \mathscr{F}}{8 \pi G}\left(H^{2}+\frac{K}{a^{2}}\right)=\left(\widehat{\rho}_{d}+\rho_{m}\right) V,
$$

where $V=(4 / 3) \pi \widetilde{r}_{A}^{3}$ is the volume of 3-dimensional sphere. Taking the time derivative of (20), we find

$$
\begin{aligned}
d \widehat{E}= & -4 \pi \widetilde{r}_{A}^{3} H\left(\hat{\rho}_{d}+\widehat{p}_{d}+\rho_{m}+p_{m}\right) d t \\
& +4 \pi \widetilde{r}_{A}^{2}\left(\hat{\rho}_{d}+\rho_{m}\right) d \widetilde{r}_{A}+\frac{\widetilde{r}_{A}}{2 G} d \mathscr{F} .
\end{aligned}
$$

Using (19) and (21) leads to

$$
\begin{aligned}
T_{h} d \widehat{S}= & d \widehat{E}+2 \pi \widetilde{r}_{A}^{2}\left(\widehat{\rho}_{d}+\rho_{m}-\widehat{p}_{d}-p_{m}\right) d \widetilde{r}_{A} \\
& +\frac{\widetilde{r}_{A}}{2 G}\left(1+2 \pi \widetilde{r}_{A} T_{h}\right) d \mathscr{F} .
\end{aligned}
$$

By introducing the work density $\widehat{W}=(1 / 2)\left(\widehat{\rho}_{d}+\rho_{m}-\widehat{p}_{d}-p_{m}\right)$ [59], one can rewrite (22) as follows:

$$
T_{h} d \widehat{S}=-d \widehat{E}+\widehat{W} d V+\frac{\widetilde{r}_{A}}{2 G}\left(1+2 \pi \widetilde{r}_{A} T_{h}\right) d \mathscr{F} .
$$


The above equation consists of additional term which is produced due to the nonequilibrium representation of thermodynamics. Consequently, the first law of thermodynamics can be expressed as follows:

$$
T_{h} d \widehat{S}+T_{h} d_{i} \widehat{S}=-d \widehat{E}+\widehat{W} d V
$$

where we have defined an entropy production term as

$$
\begin{aligned}
d_{i} \widehat{S} & =-\frac{\widetilde{r}_{A}}{2 G T_{h}}\left(1+2 \pi \widetilde{r}_{A} T_{h}\right) d \mathscr{F} \\
& =\frac{6 \pi}{G T}\left(\frac{\dot{T}+8 H T}{\dot{T}+4 H T}\right) d \mathscr{F} .
\end{aligned}
$$

The appearance of the additional term $d_{i} \widehat{S}$ illustrates that the horizon thermodynamics is nonequilibrium one in the case of nonminimal $f(T)$ gravity. Indeed, the violation of the standard first law of thermodynamics in this case is a result of the definition of the dark energy momentum components as $\widehat{\rho}_{d}$ and $\widehat{p}_{d}$, which do not satisfy the continuity equation (13). In the next section, we show that, by definition of the energy density and pressure of this generalised $f(T)$ scenario in a way that the new components satisfy the continuity equation, it is possible to have an equilibrium description of thermodynamics, so the first law of thermodynamics can be justified.

3.2. Second Law of Thermodynamics. In this subsection, we investigate the validity of the second law of thermodynamics at the apparent horizon in the framework of the nonminimal torsion-matter coupling model. The second law of thermodynamics states that the sum of the horizon entropy and the entropy of the matter field, that is, the total entropy, is a nondecreasing function of time. Assuming same temperature between the outside and inside of the apparent horizon, the condition to satisfy the second law of thermodynamics is given by

$$
\frac{d \widehat{S}}{d t}+\frac{d\left(d_{i} \widehat{S}\right)}{d t}+\frac{d \widehat{S}_{t}}{d t} \geq 0,
$$

where $d \widehat{S}$ and $d_{i} \widehat{S}$ are deduced from the first law of thermodynamics (see (24)) and $d \widehat{S}_{t}$ can be extracted from Gibb's equation which relates the entropy of all matter and energy sources to the pressure in the horizon, so that

$$
T_{h} d \widehat{S}_{t}=d\left(\widehat{\rho}_{t} V\right)+\widehat{p}_{t} d V=V d \widehat{\rho}_{t}+\left(\widehat{\rho}_{t}+\widehat{p}_{t}\right) d V,
$$

where $T_{h}$ and $\widehat{S}_{t}$ are corresponding to the temperature and entropy of total energy inside the horizon, respectively, and we have defined $\widehat{\rho}_{t} \equiv \rho_{m}+\widehat{\rho}_{d}$ and $\widehat{p}_{t} \equiv p_{m}+\widehat{p}_{d}$. Taking the time derivative of (27) and using (13) and (14), one can get

$$
T_{h} \frac{d \widehat{S}_{t}}{d t}=4 \pi \widetilde{r}_{A}^{2}\left(\hat{\rho}_{t}+\widehat{p}_{t}\right)\left(\dot{\vec{r}}_{A}-1\right)+\frac{\widetilde{r}_{A}}{2 G} \dot{\mathscr{F}} .
$$

Now, substituting the above equation and (24) in (26), we find

$$
\frac{1}{2 G} \frac{\dot{H}^{2} \mathscr{F}}{H^{4}} \geq 0 .
$$

This result describes the validity of the second law of thermodynamics in the nonequilibrium treatment. So the condition needed to hold the second law of thermodynamics in nonminimal $f(T)$ gravity is equivalent to $\mathscr{F} \geq 0$. Note that $\mathscr{F}$ should be positive in order to $\widehat{E} \geq 0$. This condition imposes a constraint to the coupling parameter $\lambda$, so that $\lambda F_{2}<\left(1+2 F_{1}\right) / 32 \pi G \rho_{m}$. As a result, the upper bound of lambda depends explicitly on the choices of two functions $f_{1}(T)$ and $f_{2}(T)$. In the flat FRW universe, the effective equation of state parameter is defined as [5]

$$
\omega_{\mathrm{eff}}=-\left(1+\frac{2 \dot{H}}{3 H^{2}}\right) \text {. }
$$

Here $\omega_{\text {eff }}>-1(\dot{H}<0)$ represents the quintessence phase of the universe, while $\omega_{\text {eff }}>-1(\dot{H}>0)$ is corresponding to the phantom phase. From (26), we find that, in the nonequilibrium picture, the second law of thermodynamics is satisfied in both phantom and quintessence phases of the universe evolution.

\section{Equilibrium Picture}

In this section, we investigate the possibility to have an equilibrium picture of thermodynamics in the nonminimal $f(T)$ modified gravity setup. To do this, we rewrite the Friedmann equations (7) in the following form:

$$
\begin{aligned}
H^{2} & =\frac{8 \pi G}{3}\left[1+\lambda\left(f_{2}-2 T F_{2}\right) \rho_{m}\right]-\frac{1}{6}\left(f_{1}-2 T F_{1}\right), \\
\dot{H} & =-\frac{8 \pi G\left(\rho_{m}+p_{m}\right)\left[1+\lambda\left(f_{2}-2 T F_{2}\right)\right]}{1+\mathscr{F}+4 T\left(F_{1}^{\prime}-16 \pi G \lambda \rho_{m} F_{2}^{\prime}\right)} .
\end{aligned}
$$

Equations (31) and (32) can be expressed as

$$
\begin{aligned}
3 H^{2} & =8 \pi G\left(\rho_{d}+\rho_{m}\right), \\
2 \dot{H}+3 H^{2} & =-8 \pi G\left(p_{d}+p_{m}\right),
\end{aligned}
$$

where we have defined the energy density and pressure of dark components as

$$
\begin{aligned}
\rho_{d} & \equiv \lambda \rho_{m} f_{2}-\frac{1}{16 \pi G}\left[f_{1}+T(1-\mathscr{F})\right] \\
p_{d} & \\
\equiv & \left(\rho_{m}+p_{m}\right)\left[\frac{2+2 \lambda\left(f_{2}-2 T F_{2}\right)}{1+\mathscr{F}+4 T\left(F_{1}^{\prime}-16 \pi G \lambda \rho_{m} F_{2}^{\prime}\right)}-1\right] \\
& -\lambda \rho_{m} f_{2}+\frac{1}{16 \pi G}\left[f_{1}+T(1-\mathscr{F})\right],
\end{aligned}
$$

respectively. Now, from the new definition of the dark energy density and pressure, the standard continuity equation can be retrieved as follows:

$$
\dot{\rho}_{d}+3 H\left(\rho_{d}+p_{d}\right)=0 .
$$

So, the equilibrium description of thermodynamics can be treated in the same manner as general relativity. 
4.1. First Law of Thermodynamics. In the new representation of the dark energy components, the time derivative of the dynamically apparent horizon is given by

$$
\frac{d \widetilde{r}_{A}}{d t}=4 \pi G \widetilde{r}_{A}^{3} H\left(\rho_{d}+p_{d}+\rho_{m}+p_{m}\right) .
$$

Introducing the Bekenstein-Hawking entropy as $S=A /(4 G)$, we find

$$
\frac{1}{2 \pi \widetilde{r}_{A}} d S=4 \pi \widetilde{r}_{A}^{3} H\left(\rho_{d}+p_{d}+\rho_{m}+p_{m}\right) d t .
$$

Substituting the horizon temperature $\left(T_{h}=-\left(1 / 2 \pi \widetilde{r}_{A}\right)[1-\right.$ $\left.\left.\dot{\vec{r}}_{A} / 2 H \widetilde{r}_{A}\right]\right)$ in the above equation leads to

$$
\begin{aligned}
T_{h} d S= & 4 \pi \widetilde{r}_{A}^{3} H\left(\rho_{d}+p_{d}+\rho_{m}+p_{m}\right) d t \\
& -2 \pi \widetilde{r}_{A}^{2}\left(\rho_{d}+p_{d}+\rho_{m}+p_{m}\right) d \widetilde{r}_{A} .
\end{aligned}
$$

Defining the Misner-Sharp energy as $E=\widetilde{r}_{A} /(2 G)=V\left(\rho_{d}+\right.$ $\left.\rho_{m}\right)$ in the Einstein gravity, we get

$$
\begin{aligned}
d E= & -4 \pi \widetilde{r}_{A}^{3} H\left(\rho_{d}+p_{d}+\rho_{m}+p_{m}\right) d t \\
& +4 \pi \widetilde{r}_{A}^{2}\left(\rho_{d}+\rho_{m}\right) d \widetilde{r}_{A} .
\end{aligned}
$$

With the definition of the work density as $W=\left(\rho_{d}+\rho_{m}-\right.$ $\left.p_{d}-p_{m}\right) / 2$ [59] and using (40) and (41), we obtain the following equation corresponding to the first law of equilibrium thermodynamics:

$$
T_{h} d S=-d E+W d V
$$

As a result, an equilibrium treatment of the first law of thermodynamics is obtained with a suitable definition for the energy momentum tensor components of dark energy.

One can show that the horizon entropy in the equilibrium picture has a relation with the horizon entropy in the nonequilibrium picture as

$$
d S=d \widehat{S}+d_{i} \widehat{S}+\frac{\widetilde{r}_{A}}{2 G T_{h}} d \mathscr{F}-\frac{2 \pi \dot{H}(1-\mathscr{F})}{G H^{3}} d t .
$$

Using (25) and (39), the above relation reduces to the following form:

$$
d S=\frac{1}{\mathscr{F}}\left(d \widehat{S}+\frac{\dot{H}+2 H^{2}}{\dot{H}+2 H^{2}} d_{i} \widehat{S}\right)
$$

The difference between $S$ and $\widehat{S}$ appearing in the nonminimal $f(T)$ gravity is due to $d \mathscr{F} / d T=0$. Note that, in the pure teleparallel gravity case, which corresponds to $f_{1}(T)=T$ and $\lambda=0$, we have $\widehat{S}=S$. From (44), we see that the change of the entropy $S$ in the equilibrium picture involves the information of both $d \widehat{S}$ and $d_{i} \widehat{S}$ in the nonequilibrium picture.

4.2. Second Law of Thermodynamics. In this subsection, we use the first law of thermodynamics in the equilibrium picture (see (42)) to find the general condition which is needed to hold the second law of thermodynamics in the nonminimal $f(T)$ scenario. As before, we assume the same temperature for outside and inside the apparent horizon of the universe. To examine the validity of the second law of thermodynamics, we consider the Gibbs relation in terms of all matter and energy components:

$$
T_{h} d S_{t}=V d \rho_{t}+\left(\rho_{t}+p_{t}\right) d V
$$

where $\rho_{t} \equiv \rho_{m}+\rho_{d}$ and $p_{t} \equiv p_{m}+p_{d}$. Using (32) and (40), the evolution of the horizon entropy is given by

$$
\dot{S}=8 \pi^{2} H \widetilde{r}_{A}^{4}\left(\rho_{t}+p_{t}\right)=\frac{6 \pi}{G} \frac{\dot{T}}{T^{2}} .
$$

To obey the second law of thermodynamics in the equilibrium picture of nonminimal $f(T)$ gravity, we require that $d S / d t+d S_{t} / d t \geq 0$. Plugging (45) and (46) into this condition, we find

$$
-(4 H T+\dot{T})=12 H\left(2 H^{2}+\dot{H}\right) \geq 0 .
$$

It is clear that this condition naturally holds in the phantom phase of the universe, where $\dot{H}>0$. On the other hand, for the validity of the second law in the quintessence phase $(\dot{H}<$ 0 ), it is required that $\dot{H}<2 H^{2}$. Note that the result (47) is independent of the forms of two functions $f_{i}(T)$ and imposes no constraint to the coupling parameter $\lambda$.

\section{Conclusions}

In this paper, we have studied the laws of thermodynamics in a generalized teleparallel gravity which contains nonminimal coupling between the matter field and an arbitrary function of the torsion scalar. From the Friedmann equations, we have constructed the first law of thermodynamics on the apparent horizon in two different approaches. These approaches depend on the definition of the components of the energy momentum tensor of dark energy and lead to the nonequilibrium and equilibrium descriptions of thermodynamics. We have seen that an entropy production term appears in the nonequilibrium description due to the violation of the standard continuity equation because of the definition of the dark energy components. Consequently, the usual first law of thermodynamics is violated in the nonequilibrium picture. We also have examined the second law of thermodynamics which illustrates that the total entropy evolution with time including the horizon entropy, the nonequilibrium entropy production term, and the entropy of all matter field and energy components is a nondecreasing function of time. We have found that the second law of thermodynamics is satisfied in both phantom and quintessence phases of the universe evolution. It should be noted that the validity of the second law of thermodynamics in the nonequilibrium picture imposes a constraint on the coupling parameter $\lambda$; that is, an upper bound on $\lambda$ can be deduced, which depends explicitly on the choices of functions $f_{1}(T)$ and $f_{2}(T)$.

To have an equilibrium description of thermodynamics, we have redefined the dark energy density and pressure in a manner that the new components satisfy the continuity 
equation, so there are no extra entropy terms and the first law of thermodynamics holds. We have also shown that the change of the horizon entropy in the equilibrium picture involves the information of the change of the horizon entropy as well as the change of the entropy production term in the nonequilibrium description.

In studying the second law of thermodynamics, we have examined the evolution of the entropy contributed by the horizon entropy and all matter fields and energy contents in the equilibrium description. We have found the condition to satisfy the second law of thermodynamics which holds in the phantom phase of the universe. Furthermore, for the validity of the second law in the nonphantom phase it is required that $\dot{H}<2 H^{2}$ and there is no constraint to the coupling parameter of the nonminimal $f(T)$ gravity scenario.

\section{Competing Interests}

The authors declare that there are no competing interests regarding the publication of this paper.

\section{Acknowledgments}

The authors would like to acknowledge Professor Kourosh Nozari for invaluable remarks.

\section{References}

[1] A. Einstein, "Riemannian geometry, while maintaining the notion of teleparallelism," Sitzungsberichte der Preussischen Akademie der Wissenschaften, vol. 17, pp. 217-221, 1928.

[2] K. Hayashi and T. Shirafuji, "New general relativity," Physical Review D, vol. 19, no. 12, pp. 3524-3553, 1979.

[3] S. Capozziello, V. F. Cardone, S. Carloni, and A. Troisi, "Curvature quintessence matched with observational data," International Journal of Modern Physics D, vol. 12, no. 10, pp.1969-1982, 2003.

[4] S. Nojiri and S. D. Odintsov, "Modified $f(R)$ gravity consistent with realistic cosmology: from a matter dominated epoch to a dark energy universe," Physical Review D, vol. 74, no. 8, Article ID 086005, 2006.

[5] S. Nojiri and S. D. Odinstsov, "Introduction to modified gravity and gravitational alternative for dark energy," International Journal of Geometric Methods in Modern Physics, vol. 4, no. 1, p. 115, 2007.

[6] T. P. Sotiriou and V. Faraoni, " $f(R)$ theories of gravity," Reviews of Modern Physics, vol. 82, no. 1, pp. 451-497, 2010.

[7] K. Nozari and T. Azizi, "Phantom-like behavior in $\mathrm{f}(\mathrm{R})$-gravity," Physics Letters B, vol. 680, no. 3, pp. 205-211, 2009.

[8] R. Ferraro and F. Fiorini, "Modified teleparallel gravity: inflation without an inflaton," Physical Review D, vol. 75, no. 8, Article ID 084031, 2007.

[9] G. R. Bengochea and R. Ferraro, "Dark torsion as the cosmic speed-up," Physical Review D, vol. 79, no. 12, Article ID 124019, 5 pages, 2009.

[10] E. V. Linder, "Einstein's other gravity and the acceleration of the Universe," Physical Review D, vol. 81, Article ID 127301, 2010.

[11] M. H. Daouda, M. E. Rodrigues, and M. J. S. Houndjo, "New static solutions in $f(T)$ theory," European Physical Journal $C$, vol. 71, article 1817, 2011.
[12] K. Bamba, C. Geng, C. Lee, and L. Luo, "Equation of state for dark energy in $f(T)$ gravity," Journal of Cosmology and Astroparticle Physics, vol. 2011, article no. 021, 2011.

[13] J. B. Dent, S. Dutta, and E. N. Saridakis, " $f(T)$ gravity mimicking dynamical dark energy. Background and perturbation analysis," Journal of Cosmology and Astroparticle Physics, vol. 2011, 2011.

[14] Y. Cai, S. Capozziello, M. D. Laurentis, and E. N. Saridakis, " $f(T)$ teleparallel gravity and cosmology," Reports on Progress in Physics, vol. 79, no. 10, Article ID 106901, 2016.

[15] T. Harko, F. S. N. Lobo, G. Otalora, and E. N. Saridakis, "Nonminimal torsion-matter coupling extension of $f(T)$ gravity," Physical Review D, vol. 89, no. 12, Article ID 124036, 2014.

[16] C. J. Feng, F. Ge, X. Li, R. Lin, and X. Zhai, "Towards realistic $f(T)$ models with nonminimal torsion-matter coupling extension," Physical Review D, vol. 92, no. 10, Article ID 104038, 2015.

[17] S. Carloni, F. S. Lobo, G. Otalora, and E. N. Saridakis, "Dynamical system analysis for a nonminimal torsion-matter coupled gravity," Physical Review D, vol. 93, no. 2, Article ID 024034, 2016.

[18] S. W. Hawking, "Particle creation by black holes," Communications in Mathematical Physics, vol. 43, no. 3, pp. 199-220, 1975.

[19] J. D. Bekenstein, "Black holes and entropy," Physical Review. D, vol. 7, pp. 2333-2346, 1973.

[20] T. Jacobson, "Thermodynamics of spacetime: the Einstein equation of state," Physical Review Letters, vol. 75, no. 7, pp. 12601263, 1995.

[21] R.-G. Cai and S. P. Kim, "First law of thermodynamics and Friedmann equations of Friedmann-Robertson-Walker universe," Journal of High Energy Physics, vol. 2005, 2005.

[22] M. Akbar and R. Cai, "Thermodynamic behavior of the Friedmann equation at the apparent horizon of the FRW universe," Physical Review D, vol. 75, no. 8, Article ID 084003, 2007.

[23] R.-G. Cai and L.-M. Cao, "Unified first law and the thermodynamics of the apparent horizon in the FRW universe," Physical Review D, vol. 75, no. 6, Article ID 064008, 2007.

[24] R.-G. Cai, L.-M. Cao, Y.-P. Hu, and S. Kim, "Generalized Vaidya spacetime in Lovelock gravity and thermodynamics on the apparent horizon," Physical Review D, vol. 78, no. 12, Article ID 124012, 2008.

[25] A. Sheykhi, B. Wang, and R.-G. Cai, "Deep connection between thermodynamics and gravity in Gauss-Bonnet braneworlds," Physical Review D, vol. 76, no. 2, Article ID 023515, 5 pages, 2007.

[26] S.-F. Wu, B. Wang, G.-H. Yang, and P.-M. Zhang, "The generalized second law of thermodynamics in generalized gravity theories," Classical and Quantum Gravity, vol. 25, no. 23, Article ID 235018, 2008.

[27] C. Eling, R. Guedens, and T. Jacobson, "Nonequilibrium thermodynamics of spacetime," Physical Review Letters, vol. 96, no. 12, Article ID 121301, 2006.

[28] K. Bamba and C.-Q. Geng, "Thermodynamics in $f(R)$ gravity in the Palatini formalism," Journal of Cosmology and Astroparticle Physics, vol. 2010, no. 6, article 014, 20 pages, 2010.

[29] K. Bamba, C.-Q. Geng, and S. Tsujikawa, "Equilibrium thermodynamics in modified gravitational theories," Physics Letters B, vol. 688, no. 1, pp. 101-109, 2010.

[30] K. Bamba and C. Geng, "Thermodynamics of cosmological horizons in $f(T)$ gravity," Journal of Cosmology and Astroparticle Physics, vol. 2011, article no. 008, 2011.

[31] K. Karami, M. S. Khaledian, and N. Abdollahi, "The generalized second law of gravitational thermodynamics on the apparent horizon in $f(R)$-gravity," $E P L$, vol. 98, no. 3, 2012. 
[32] K. Bamba, C. Geng, S. Nojiri, and S. D. Odintsov, "Equivalence of the modified gravity equation to the Clausius relation," $E P L$ (Europhysics Letters), vol. 89, no. 5, p. 50003, 2010.

[33] M. Sharif and M. Zubair, "Thermodynamics in $f(R, T)$ theory of gravity," Journal of Cosmology and Astroparticle Physics, vol. 3, article 28, 2012.

[34] T. Harko, "Thermodynamic interpretation of the generalized gravity models with geometry-matter coupling," Physical Review D, vol. 90, no. 4, Article ID 044067, 2014.

[35] M. Sharif and M. Zubair, "Thermodynamics in $f(R, T)$ theory of gravity," Journal of Cosmology and Astroparticle Physics, vol. 2012, no. 3, article 028, 2012.

[36] M. Sharif and M. Zubair, "Thermodynamics in modified gravity with curvature matter coupling," Advances in High Energy Physics, vol. 2013, Article ID 947898, 10 pages, 2013.

[37] T. Azizi and N. Borhani, "Thermodynamics in hybrid metricPalatini gravity," Astrophysics and Space Science, vol. 357, article 146, 2015.

[38] R. Miao, M. Li, and Y. Miao, "Violation of the first law of black hole thermodynamics in $f(T)$ gravity," Journal of Cosmology and Astroparticle Physics, vol. 2011, 2011.

[39] K. Karami and A. Abdolmaleki, "Generalized second law of thermodynamics in $f(T)$ gravity," Journal of Cosmology and Astroparticle Physics, vol. 2012, no. 4, article 007, 2012.

[40] K. Bamba and C. Geng, "Thermodynamics of cosmological horizons in $f(T)$ gravity," Journal of Cosmology and Astroparticle Physics, vol. 2011, 2011.

[41] M. Sharif and S. Rani, "Thermodynamics in $f(T)$ gravity and corrected entropies," The European Physical Journal Plus, vol. 128, article 96, 2013.

[42] M. Zubair and S. Waheed, "Energy conditions in $\mathrm{f}(\mathrm{T})$ gravity with non-minimal torsion-matter coupling," Astrophysics and Space Science, vol. 355, no. 2, pp. 361-369, 2015.

[43] M. Zubair and S. Waheed, "Thermodynamic study in modified $f(T)$ gravity with cosmological constant regime," Astrophysics and Space Science, vol. 360, article 68, 2015.

[44] M. Zubair and A. Jawad, "Generalized second law of thermodynamics in $f\left(T, T_{G}\right)$ gravity," Astrophysics and Space Science, vol. 360, no. 1, article 11, 2015.

[45] M. Askin, H. Abedi, and M. Salti, "Thermodynamics in $\mathrm{f}(\mathrm{T}, \theta)$ gravity," Romanian Journal of Physics, vol. 60, no. 1-2, pp. 4-55, 2015.

[46] P. C. Davies, "Cosmological horizons and the generalised second law of thermodynamics," Classical and Quantum Gravity, vol. 4, no. 6, pp. L225-L228, 1987.

[47] H. M. Sadjadi, "Generalized second law in a phantom-dominated universe," Physical Review D, vol. 73, no. 6, 2006.

[48] M. R. Setare and S. Shafei, "A holographic model of dark energy and the thermodynamics of a non-flat accelerated expanding universe," Journal of Cosmology and Astroparticle Physics, vol. 2006, article no. 011, 2006.

[49] E. Babichev, V. Dokuchaev, and Y. Eroshenko, "Black hole mass decreasing due to phantom energy accretion," Physical Review Letters, vol. 93, no. 2, Article ID 021102, 2004.

[50] J. Zhou, B. Wang, Y. Gong, and E. Abdalla, "The generalized second law of thermodynamics in the accelerating universe," Physics Letters. B, vol. 652, no. 2-3, pp. 86-91, 2007.

[51] G. Izquierdo and D. Pavón, "The generalized second law in phantom dominated universes in the presence of black holes," Physics Letters B, vol. 639, no. 1, pp. 1-4, 2006.
[52] M. Jamil and M. Akbar, "Generalized second law of thermodynamics for a phantom energy accreting BTZ black hole," General Relativity and Gravitation, vol. 43, no. 4, pp. 1061-1068, 2011.

[53] A. Abdolmaleki, T. Najafi, and K. Karami, "Generalized second law of thermodynamics in scalar-tensor gravity," Physical Review D, vol. 89, no. 10, Article ID 104041, 2014.

[54] S. Wu, B. Wang, G. Yang, and P. Zhang, "The generalized second law of thermodynamics in generalized gravity theories," Classical and Quantum Gravity, vol. 25, no. 23, 2008.

[55] J. M. Bardeen, B. Carter, and S. W. Hawking, "The four laws of black hole mechanics," Communications in Mathematical Physics, vol. 31, pp. 161-170, 1973.

[56] R. M. Wald, "Black hole entropy is the Noether charge," Physical Review D, vol. 48, no. 8, pp. R3427-R3431, 1993.

[57] R. Brustein and M. Hadad, "Einstein equations for generalized theories of gravity and the thermodynamic relation $\delta Q=T \delta S$ are equivalent," Physical Review Letters, vol. 103, no. 10, Article ID 101301, 4 pages, 2009.

[58] B. Li, T. P. Sotiriou, and J. D. Barrow, "Large-scale structure in f(T) gravity," Physical Review D, vol. 83, no. 10, 2011.

[59] S. A. Hayward, "Unified first law of black-hole dynamics and relativistic thermodynamics," Classical and Quantum Gravity, vol. 15, no. 10, pp. 3147-3162, 1998. 

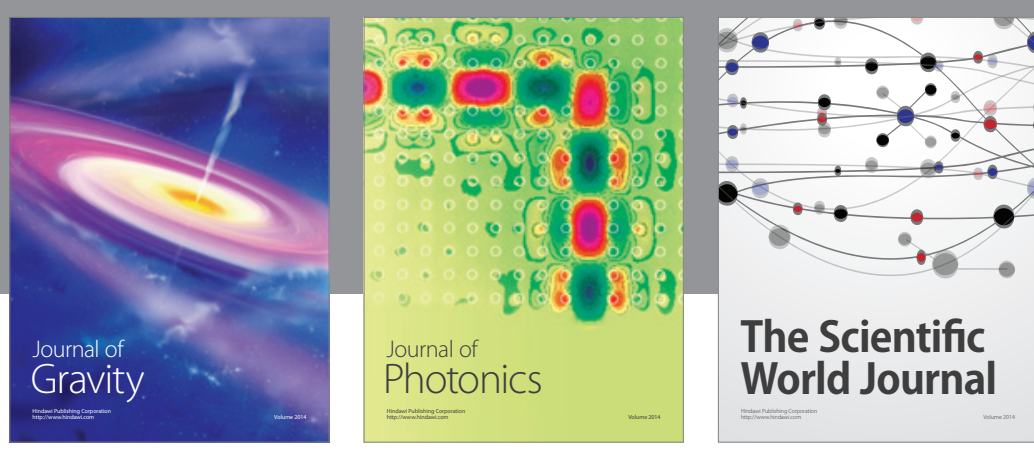

The Scientific World Journal
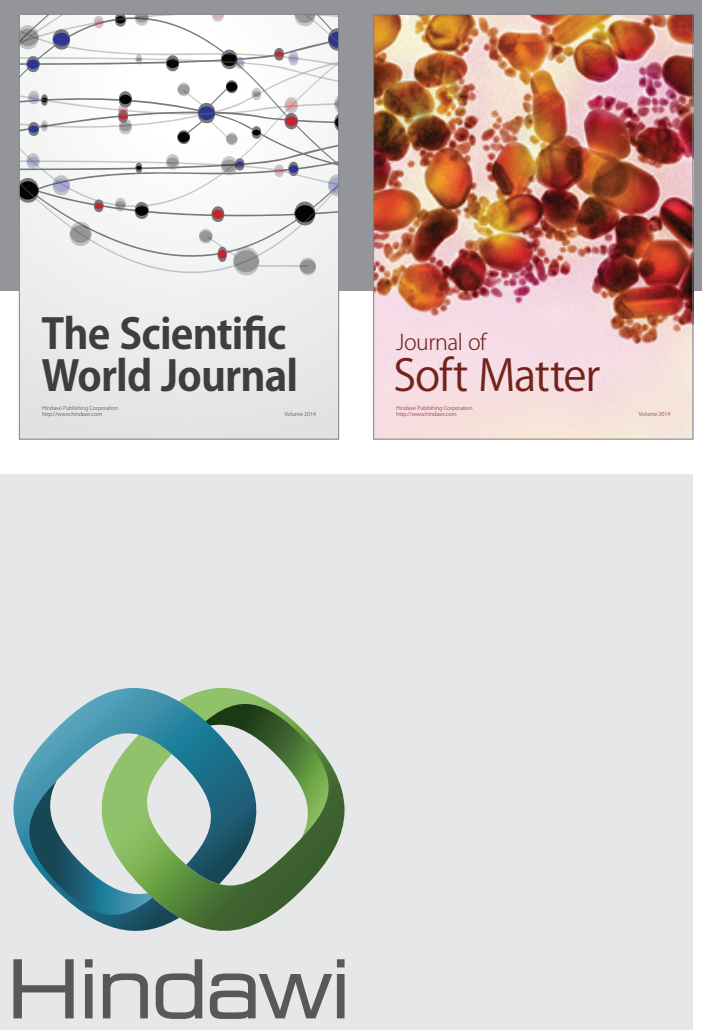

Submit your manuscripts at

https://www.hindawi.com
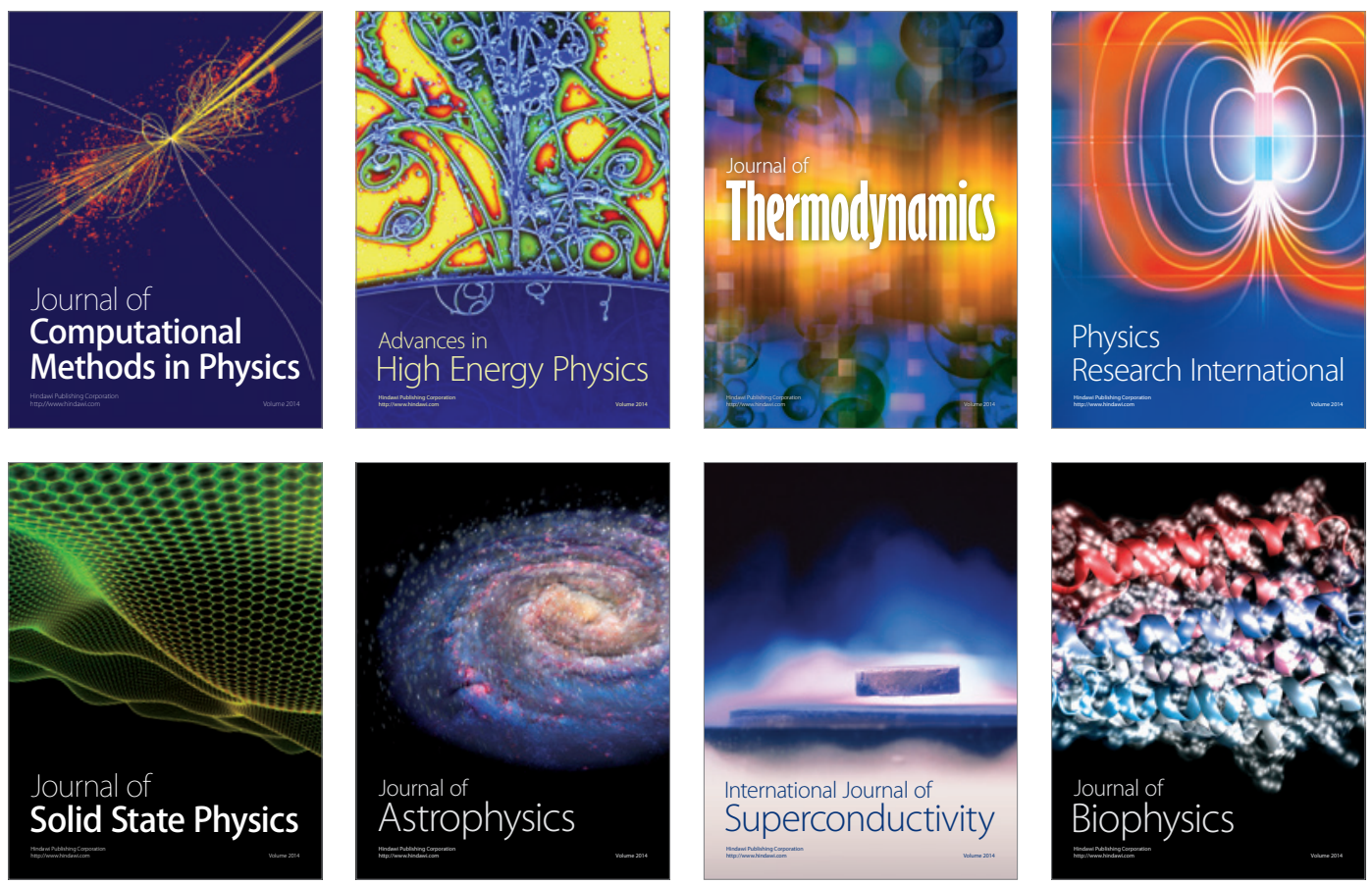
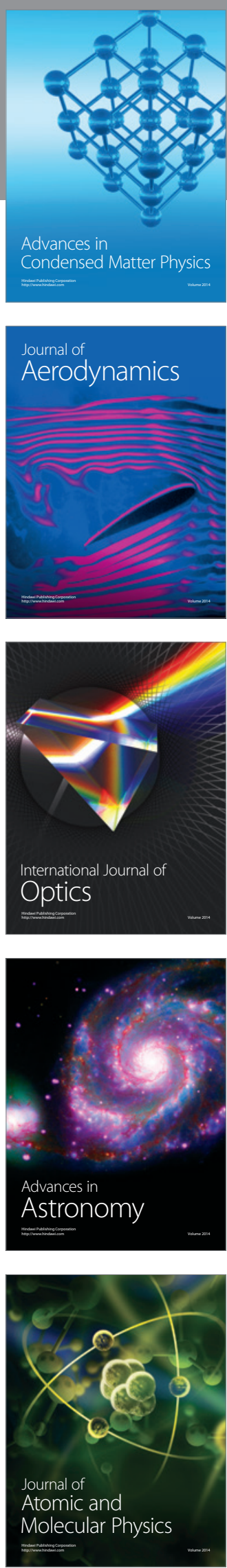